\title{
Neue Behandlungsmöglichkeiten für Epilepsie
}

\author{
M. Wagner
}

Etwa 0,5 bis $2 \%$ aller Menschen leiden unter Epilepsie. Diese Krankheit kann, unabhängig von Familiengeschichte, Gesundheitsvorsorge und sozialer Herkunft, jeden in jedem Alter treffen. Allein in der Schweiz leben etwa 70000 Epileptiker, und jedes Jahr kommen etwa 4000 hinzu. Eine Epilepsieerkrankung kann für die Betroffenen verheerende Auswirkungen haben - bei richtigem Umgang mit der Krankheit wird die Lebensqualität jedoch meist kaum eingeschränkt. In den letzten Jahren ergaben sich neue Optionen für den Umgang mit Epilepsie. Neue antiepileptische Wirkstoffe kamen auf den Markt, es besteht die Möglichkeit der Vagusnervstimulation, und es gibt Fortschritte im Bereich der Chirurgie.

In diesem Beitrag soll zunächst ein Überblick über die neueren Entwicklungen in der Epilepsiebehandlung gegeben werden. Der Schwerpunkt soll dann in der Beschreibung einer neuen Technik zur verbesserten EEG-gestützten Operationsplanung liegen, mit der sich unsere Arbeitsgruppe beschäftigt. Um die Relevanz dieser neuen Technik einordnen zu können, erscheint es jedoch sinnvoll, zunächst die anderen Therapiemöglichkeiten zu streifen.

\section{Antiepileptische Wirkstoffe}

Die Verwendung antiepileptischer Pharmazeutika (AEP; Tab. 1) ist das Hauptfeld der Epilepsiebehandlung. Die konventionellen AEPs, darunter Carbamazepin, Valproinsäure, Phenytoin, Phenobarbital und Primidon, sind die meistverschriebenen. Ethosuximid ist ein traditionelles AEP, welches ausschliesslich bei Absenzen verwendet wird. Die Verwendung von AEPs war und bleibt problematisch, da das therapeutische Fenster - definiert durch die Dosierung, häufige dosierungsabhängige Nebenwirkungen auf das ZNS und seltener vorkommende schwere Vergif-

tungen - oft recht eng ist. Aus diesem Grund wurde verstärkt nach AEPs gesucht, die besser verträglich, sicherer und wirksamer sind.

Die neue Ära begann 1993 in den USA mit der Genehmigung von Felbamat, welches das erste neugenehmigte AEP seit 1978 (Valproinsäure) war. Die anfängliche Begeisterung für den neuen Wirkstoff erstarb, als - nachdem etwa $100000 \mathrm{~Pa}-$ tienten ihn eingenommen hatten - 35 Fälle aplastischer Anämie und 18 Fälle von Hepatitis bekannt wurden. Seitdem wird das Medikament nurmehr bei refraktorischer Epilepsie eingesetzt, einem Krankheitsbild, bei dem die möglichen Vorteile die Risiken noch überwiegen. Der Damm war jedoch gebrochen, und seitdem wurden mehrere neue Wirkstoffe eingeführt, nämlich Vigabatrin, Lamotrigin, Gabapentin, Tiagabin und zuletzt Topiramat. Diese wurden zunächst nur zur Zusatzbehandlung bei schwer behandelbaren Epilepsien zugelassen. Lamotrigin, Gabapentin und Topiramat haben auch die Zulassung zur Monotherapie.

\section{Anfälle fokalen Ursprungs}

Anfälle partiellen bzw. fokalen Ursprungs sind bei Erwachsenen am häufigsten. Daher wurden alle traditionellen und neuen AEPs für diese Krankheitsbilder getestet und, zumindest als $\mathrm{Zu}$ satzmedikation, auch genehmigt. Es gibt noch

Korrespondenz:

Dr. Michael Wagner

Compumedics / Neuroscan

Heussweg 25

D-20255 Hamburg

E-Mail: mwagner@neuroscan.com
Tabelle 1

Antiepileptische Pharmazeutika.

\begin{tabular}{ll} 
Wirkstoff & Produkte (alle $\left.{ }^{\circledR}\right)$ \\
\hline Carbamazepin & Finlepsin, Fokalepsin, Sirtal, Timonil, Tegretal \\
\hline Valproinsäure & Convulex, Convulsofin, Ergenyl, Leptilan, Mylproin, Orfiril, Depakote \\
\hline Phenytoin & Epanutin, Phenyhydan, Phenytoin AWD, Zentropil, Dilantin \\
\hline Phenobarbital & Lepinal, Lepinaletten, Luminal, Luminaletten, Phenaemal, Phenaemaletten \\
\hline Primidon & Lepsiral, Liskantin, Mylepsinum, Resimatil \\
\hline Ethosuximid & Petnidan, Suxilep, Suxinutin \\
\hline Felbamat & Taloxa, Felbatol \\
\hline Vigabatrin & Sabril \\
\hline Lamotrigin & Lamictal \\
\hline Gabapentin & Neurontin \\
\hline Tiagabin & Gabitril \\
\hline Topiramat & Topamax \\
\hline
\end{tabular}


keine direkte Vergleichsstudie der neuen AEPs untereinander, aber alle scheinen für fokale Anfälle ähnlich effektiv zu sein. Dies bedeutet, dass sie bei 30 bis $40 \%$ aller Patienten die Anfallshäufigkeit auf weniger als die Hälfte reduzieren, wenn sie zusätzlich zu denjenigen AEPs eingenommen werden, die der Patient schon verwendet. Die Studien zeigen einerseits, dass kein einzelnes AEP bei allen wirkt, aber auch, dass bei Patienten, die auf ein bestimmtes Mittel nicht ansprechen, ein anderes sehr wirksam sein kann. Die Faktoren, die bestimmen, welches AEP bei wem wirkt, sind noch unbekannt.

Alle bis jetzt für Anfälle fokalen Ursprungs als Zusatzmedikation getesteten Mittel sind auch als initiale Monotherapie für solche Anfälle wirksam und einige sind hierfür auch schon zugelassen. Die meisten Ärzte beginnen die Behandlung jedoch mit einem der traditionellen AEPs, da deren Nebenwirkungen besser abschätzbar sind und weil sie kostengünstiger sind. Es darf vermutet werden, dass - mit wachsender Erfahrung mit den neuen Mitteln - diese die traditionellen AEPs auch mehr und mehr im Bereich der Initialtherapie verdrängen werden.

\section{Generalisierte Anfälle}

Generalisierte Anfälle sind die häufigste Epilepsieart bei Kindern. Diese Anfallsklasse beinhaltet Absenzen («petit mal»), primäre generalisierte tonisch-klonische («grand mal»), myoklonische und atonische Anfälle. Absenzen können meist mit Valproinsäure und Ethosuximid behandelt werden. Das Lennox-Gastaut-Syndrom ist eine refraktorische Art der Epilepsie, bei der verschiedene Arten von Anfällen, Nachlassen der mentalen Fähigkeiten und charakteristische Veränderungen im EEG einhergehen. Für diese Krankheit hat sich Valproinsäure als das effektivste traditionelle AEP erwiesen; Lamatrigin und Topiramat sind ebenfalls wirksam. Auch bei Felbamat überwiegt der Nutzen im Fall von Lennox-Gastaut meist die Risiken.

\section{Auswahl des geeigneten AEPs}

Die Menge heute verfügbarer AEPs macht die Auswahl schwer. Verschiedene Grundregeln werden dabei angewendet und gelten sowohl für die traditionellen als auch für die neuen Medikamente:

1. Auswahl nach Anfallsart und -syndrom;

2. für Anfälle fokalen Ursprungs: Auswahl nach pharmakokinetischen Kriterien, Dosierungshäufigkeit und Kosten, da praktisch alle AEPs ausser Ethosuximid hier Erfolg versprechen;
3. zunächst nur ein AEP verwenden und nie mehr als eines zur Zeit hinzunehmen;

4. mit niedrigen Dosierungen beginnen und nur langsam steigern, da die im Beipackzettel empfohlene Steigerungsrate nur für ansonsten gesunde Patienten, die nur ein AEP zur Zeit und keine psychoaktiven Substanzen einnehmen, gilt;

5. die Dosis solange steigern, bis das Mittel wirkt oder bis sich bei Erreichen der Maximaldosis bzw. anhand der einsetzenden Nebenwirkungen erweist, dass das Mittel nicht hilft.

\section{Vagusnervstimulation}

Die elektrische Stimulation des linken N. Vagus (auch Vagusstimulation oder VNS genannt) wurde 1994 in Europa und 1997 in den USA als Zusatztherapie für partielle Epilepsie zugelassen. Es konnte nachgewiesen werden, dass die Anfallshäufigkeit nach 3 Monaten bei $30 \%$ und nach 18 Monaten bei $50 \%$ aller Patienten auf weniger als die Hälfte zurückging. Die genaue Wirkungsweise der Vagusnervstimulation ist unbekannt; die Methode lindert auch Depressionen.

Zur VNS wird ein Signalerzeuger im linken oberen Brustkorb implantiert (ähnlich wie z.B. ein Herzschrittmacher) und mit einer bipolaren Stimulationselektrode verbunden, welche im Halsbereich mit dem linken Vagusnerv verbunden wird. Zur Programmierung des Signalgebers wird ein berührungsloser Transmitter, der an einen tragbaren Computer angeschlossen ist, vor die Brust gehalten. Mittels spezieller Magnete können der Patient oder Angehörige den Signalgeber aktivieren, um einen Anfall zu stoppen oder zu verkürzen. Nebenwirkungen der VNS sind Heiserkeit, Husten und Dyspnoe, woran sich die Patienten allerdings erstaunlich schnell gewöhnen.

Ein Nachteil der Methode ist, dass - auch wenn die Stimulation keine Wirkung zeigt - die Elektroden nicht entfernt werden, da deren Entfernung mit einem höherem Risiko verbunden ist als ihr Belassen. Der Signalgeber kann allerdings wieder entfernt werden, wobei eine kleine Narbe zurückbleibt.

\section{Epilepsieoperationen}

Die Operation wurde früher als letztes Mittel betrachtet, neue Verfahren machen sie jedoch sicher und effektiv, und sie ist die einzige heute bekannte Methode, die die Epilepsie tatsächlich zu heilen vermag. Eine Operation sollte früh- 
zeitig erwogen werden, um die möglichen negativen Folgen einer schlecht kontrollierten Epilepsie zu minimieren. Sie sollte dann in Betracht gezogen werden, wenn die Monotherapie mit zwei verschiedenen erfolgversprechenden Substanzen nicht angeschlagen hat, da in solch einem Fall die Wahrscheinlichkeit, mit einem anderen Mittel die Krankheit unter Kontrolle zu bekommen, weniger als $20 \%$ beträgt. Hierzu muss nachgewiesen sein, dass die Medikamente ordnungsgemäss eingenommen wurden, und die Epilepsie muss (aufgrund der Anfallshäufigkeit oder der medikamentösen Nebenwirkungen) die Lebensqualität massiv beeinträchtigen.

\section{Operationsplanung}

Falls aufgrund dieser Voraussetzungen ein Patient von einer Operation profitieren könnte, so wird eine diagnostische Evaluierung durchgeführt, um die tatsächliche Operationseignung zu ermitteln. Diese Evaluierung beinhaltet neurologische Bildgebung, stationäre Video- und EEGDaueraufzeichnung sowie neuropsychologische Tests.

Hochauflösende kernspintomographische Aufnahmen (Magnetic Resonance Imaging, MRI) sind unerlässlich, um strukturelle Unregelmässigkeiten $\mathrm{zu}$ identifizieren, welche auf den Ursprung epileptischer Anfälle hindeuten können. Beispiele sind eine mögliche asymmetrische Verkümmerung des Temporallappens oder des Hippokampus, eine Sklerose des Hippokampus, kortikale Fehlbildungen, Gefässfehlbildungen und Tumore.

SPECT-Aufnahmen (Single Photon Emission Computed Tomography) helfen dabei, Gebiete veränderter Durchblutung im Bereich des vermuteten epileptischen Fokus zu identifizieren. Wenn die Aufnahmen zwischen Anfällen (interiktal) durchgeführt werden, so zeigt SPECT entweder gar keine Asymmetrie der Durchblutung oder eine verringerte Durchblutung im epileptischen Bereich, welche vermutlich auf verringerte neuronale Aktivität zurückzuführen ist. Für Aufnahmen während eines Anfalls (iktal) zeigt sich ein anderes Bild: Der Ort des Anfallsursprungs weist nun eine gesteigerte Durchblutung auf, welche für Gebiete neuronaler Hyperaktivität typisch ist. Die Durchführung iktaler Aufnahmen erfordert die genaue Kenntnis des Anfallsbeginns, da innerhalb von 90 Sekunden eine für die SPECT-Aufnahmen erforderliche radioaktive Substanz gespritzt werden muss. Dies ist daher nur unter stationärer Video-/EEG-Beobachtung möglich.
Auch die Positronenemissionstomographie (PET) kann den interiktal verringerten Stoffwechsel nachweisen.

Um die Anfälle aufzuzeichnen, wird eine stationäre telemetrische EEG-Überwachung in Verbindung mit Videomonitoring durchgeführt. Ziel dieser Untersuchung ist es, zu bestätigen, dass die Anfälle tatsächlich epileptischer Natur sind, dass sie von einem einzigen Ort ausgehen, sowie die Identifizierung des genauen Ursprungsortes. Es werden typischerweise drei bis vier Anfälle aufgezeichnet, häufig werden sogar noch mehr benötigt.

Wird der Ursprungsort durch die vorgenannten Methoden nicht ausreichend identifiziert, so können EEG-Elektroden implantiert werden, um den epileptischen Fokus zu lokalisieren. Die Elektroden werden am vermuteten Ort plaziert, wobei Tiefen- und Oberflächenelektroden (subdurale Gitter und Streifen) zur Verfügung stehen. Die Elektroden werden mehrere Tage getragen, um genügend Anfälle aufzuzeichnen und deren Ursprungsort zu bestimmen. Da die Verwendung implantierter Elektroden zwei Operationen verlangt (Anbringen der Elektroden sowie Entfernung der Elektroden und Resektion des epileptischen Fokus), wird intensiv nach alternativen Mitteln gesucht. Ein möglicher Weg wird von unserer Arbeitsgruppe verfolgt und soll weiter unten beschrieben werden.

Neuropsychologische Tests dienen dazu, die Lateralisierung von Sprache und Gedächtnis zu bestimmen und das Risiko operationsbedingter funktioneller Defizite einzuschätzen. Falls erwogen wird, einen Teil des Temporallappens zu entfernen, wird ein Amobarbital-Test (auch Wada-Test genannt) durchgeführt. Dieser dient dazu herauszufinden, welche Hemisphäre sprachdominant ist und ob der nach einer Operation verbleibende Schläfenlappen für den Erhalt des Gedächtnisvermögens ausreicht.

\section{Operationsarten}

Die häufigste Operationsart ist die Entfernung des vorderen Teils des Schläfenlappens, da mesial-temporale Sklerose die am häufigsten diagnostizierte einzelne mit Epilepsie verursachende Schädigung darstellt. Bei dieser Operation werden die vorderen 3 bis $3 \frac{1}{2} \mathrm{~cm}$ des Temporallappens und der mesial-temporalen Strukturen entfernt. Eine Vergleichsstudie zeigte, dass $64 \%$ aller Patienten dieses Krankheitsbilds nach einer Operation anfallsfrei wurden, im Gegensatz zu nur $8 \%$ derer mit AEP-Therapie. Erst ein Jahr nach erfolgreicher OP wird erwogen, die 
Abbildung 1

Aus kernspintomographischen Aufnahmen berechnete Grenzflächen der relevanten leitenden Bereiche des Kopfes (Haut, Knochen, Gehirn; links und oben), aus denen ein realistisch geformtes, individuelles Volumenleitermodell erstellt werden kann (rechts unten).
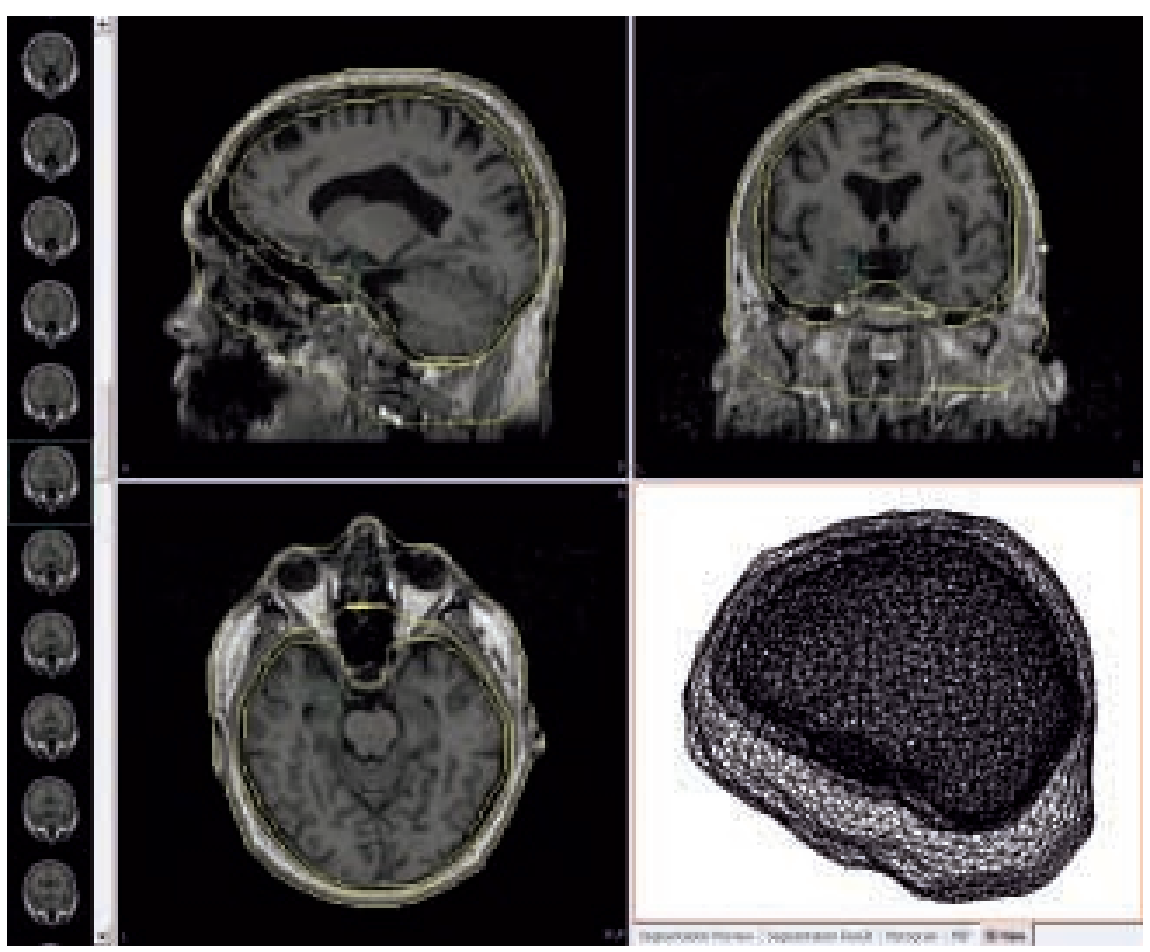

AEPs abzusetzen. Das Risiko schwerer operationsbedingter Schädigung (z.B. durch Schlaganfall) beträgt $1 \%$; 10 bis $15 \%$ aller Patienten leiden allerdings unter vorübergehenden Beeinträchtigungen (Kopfschmerzen, Depressionen, Sprach- und Gedächtnisschwächen).

Andere Resektionen haben eine etwas geringere Erfolgsquote von etwa $50 \%$, wobei jedoch die Erfolgsquote mit AEPs auch in dieser Gruppe nur $10 \%$ beträgt. Auch bei solchen Eingriffen ist die Komplikationsrate niedrig, setzt allerdings voraus, dass vorbereitend eine sorgfältige Ortung (Mapping) der wichtigen Hirnregionen (Motorkortex, Sprache) durchgeführt wird, um solche nicht mitzuentfernen. Dieses Mapping wird üblicherweise durch elektrische Stimulation mit Hilfe der implantierten Elektroden durchgeführt.

\section{EEG-Quellokalisation}

Die Verwendung implantierter Elektroden ist dann erforderlich, wenn sich die anderen Methoden nicht als trennscharf genug erweisen, um den epileptischen Fokus eindeutig zu identifizieren. So ist es z.B. nicht trivial, aus der
Betrachtung der Kurvenformen oder der Isopotential-Mappings des Oberflächen-EEGs auf die erzeugende Hirnregion zu schliessen. Neue Entwicklungen in der EEG-Quellokalisation versprechen eine derartige Genauigkeitssteigerung, dass der Einsatz implantierter Elektroden in $\mathrm{Zu}$ kunft zur seltenen Ausnahme werden könnte. So können der Diagnoseprozess beschleunigt, das Operationsrisiko verringert und die Behandlungskosten reduziert werden. Die Methode ist derzeit in klinischer Erprobung, wobei deren Ergebnisse mit denen parallel durchgeführter traditioneller Diagnostik, speziell mit Hilfe implantierter Elektroden, verglichen werden.

Die bei der Quellokalisation zu lösenden Schwierigkeiten lassen sich mit Hilfe von zwei Schlagworten beschreiben: Vorwärtsproblem und inverses Problem.

\section{Das Vorwärtsproblem}

Das Vorwärtsproblem beinhaltet die Ermittlung der EEG-Signale bei gegebener Hirnaktivität. Hierfür ist eine Beschreibung der elektrischen Leitfähigkeitsverteilung im Bereich des Kopfes erforderlich sowie eine genaue Bestimmung der Elektrodenorte. Ein Durchbruch konnte erzielt werden, als es gelang, die Leiteigenschaften des Kopfes nicht mehr mit einem Kugelmodell (wie bisher üblich), sondern mit Hilfe der Grenzelementmethode (Boundary Element Method, BEM) zu beschreiben. So ist es möglich, die individuelle Anatomie des Patienten zu modellieren. Unsere Gruppe hat Verfahren entwickelt, die aus den vorliegenden MRI-Daten die Form der relevanten Grenzflächen (Knocheninnenseite, Knochenaussenseite, Hautoberfläche) automatisch extrahieren und so ein hochauflösendes Leitfähigkeitsmodell erzeugen. Die Elektrodenorte werden mit Hilfe eines 3D-Digitalisierers bestimmt. Dabei wird jede Elektrode kurz mit einem Stift berührt und dessen Ort von einem meist optischen oder magnetischen - System registriert.

\section{Das inverse Problem}

Nun liegen aber tatsächlich die EEG-Signale vor, und die zugrundeliegende Hirnaktivität soll ermittelt werden. Die Schwierigkeit beim hierbei zu lösenden inversen Problem ist vor allem die Mehrdeutigkeit: Es sind verschiedene Gehirnzustände denkbar, welche exakt dieselben Messdaten erzeugen würden. Zudem existieren nur wenige Messorte (Elektroden), und die Messda- 


\section{Abbildung 2}

Quellanalyse eines interiktalen Spikes mittels traditionellem Kugelvolumenleiter. In dieser schematischen Ansicht macht alles noch Sinn; der Aktivitätsverlauf scheint entlang des Temporallappens zu erfolgen. Man sieht die EEG-Elektroden, das verwendete Volumenleitermodell (3-Schalen-Kugelmodell) sowie den zeitlichen und örtlichen Verlauf der rekonstruierten Hirnaktivität (Stromdipole, wobei Farbe für Zeit, Grösse für Stärke, die Kugel für den Ort und der Zylinder für die Quellorientierung stehen).

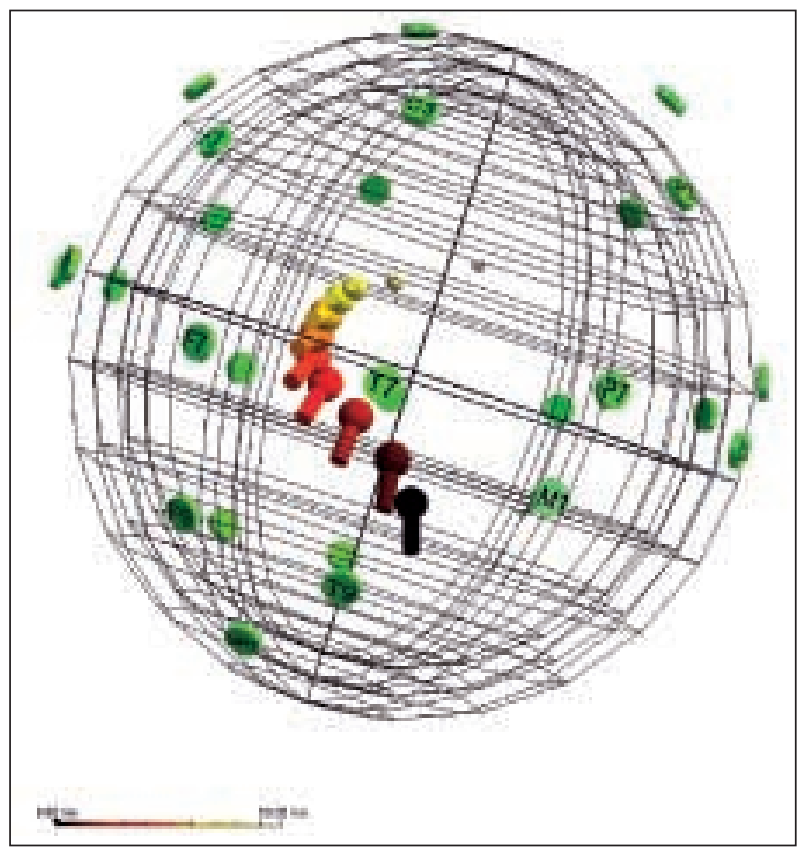

\section{Abbildung 3}

Dieselben Ergebnisse wie in Abb. 2, allerdings nun im individuellen anatomischen Kontext dargestellt. Haut und Kortex wurden aus kernspintomographischen Aufnahmen extrahiert. Man sieht deutlich die durch das falsche Volumenleitermodell verursachte schlechte Korrespondenz zwischen den Ergebnissen der Quellanalyse und der Anatomie. So sollte keine Operationsplanung durchgeführt werden!

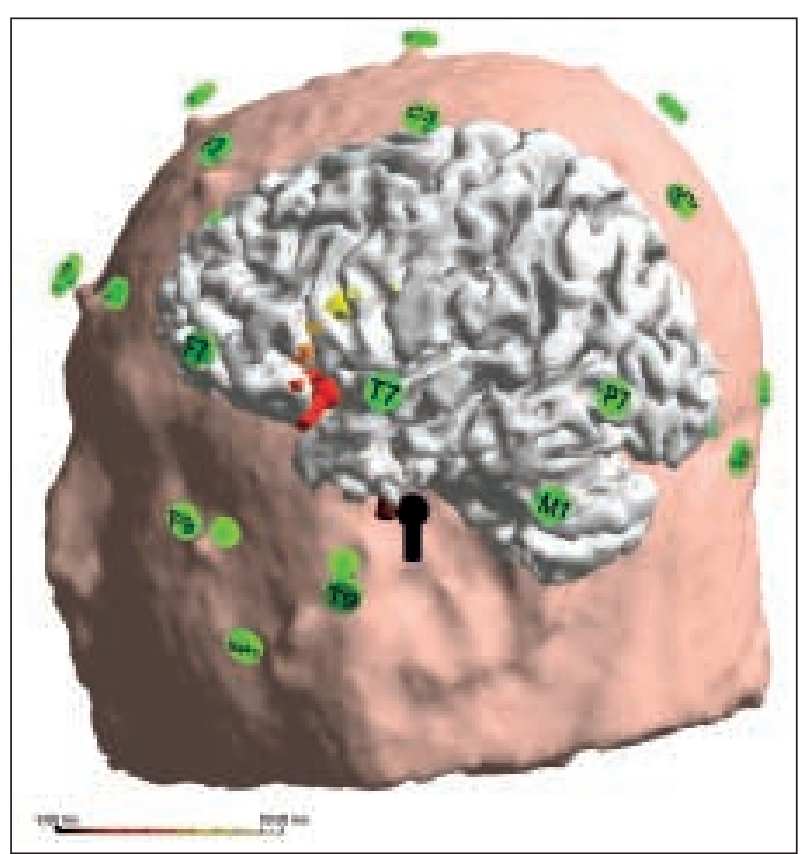

ten sind ungenau (verrauscht). Das Problem der Mehrdeutigkeit wird durch eine genauere Beschreibung der Art erwarteter Hirnaktivität umgangen. Die Art dieser genaueren Charakterisierung bestimmt den zu verwendenden Algorithmus. Erfolgversprechende Annahmen beziehen sich dabei auf die zeitliche Abfolge (sind nur eine oder wenige Hirnregionen momentan aktiv?) und den Aufbau des Kortex (senkrecht zur Kortexoberfläche ausgerichtete, stark gekoppelte Pyramidalzellen erzeugen die gemessenen Signale). Die Verwendung der in der Epilepsiediagnostik üblichen etwa 30 Elektroden ist zwar ausreichend, mit steigender Elektrodenanzahl steigt allerdings auch die Genauigkeit der inversen Verfahren.

\section{Ergebnisse und Ausblick}

Die Verwendung individueller, realistischer Volumenleitmodelle macht Lokalisationsgenauigkeiten im Millimeterbereich möglich. Zusammen mit inversen Algorithmen und sinnvollen Annahmen über die zugrundeliegende Hirnaktivität ist es möglich, den epileptischen Fokus genau genug zu lokalisieren, um ausreichende Übereinstimmung mit den Messergebnissen implantierter Elektroden zu erzielen. Es lässt sich vermuten, dass die Plazierung implantierter Elektroden mit Hilfe der Quellokalisation aus dem Oberflächen-EEG in Zukunft sehr viel zielgerichteter vorgenommen werden kann und dass es in einem nächsten Schritt möglich werden könnte, auf implantierte Elektroden in den meisten Fällen ganz zu verzichten.

\section{Fazit}

Fortschritte in der Epilepsiebehandlung ermöglichen Heilung und verbesserte Lebensqualität in einem früher ungekannten Ausmass. Der Schlüssel zu einem effektiven Epilepsiemanagement liegt in der angemessenen Nutzung dieser Fortschritte: Die pharmazeutische Strategie sollte darauf abzielen, das geeignetste AEP für den spezifischen Anfallstyp zu finden. Eine Operation sollte möglichst früh erwogen werden. Durch exakte EEG-Quellokalisierung könnte es bald möglich sein, auf implantierte Elektroden und damit auf eine vorbereitende Operation $\mathrm{zu}$ verzichten. 
Abbildung 4

Quellanalyse eines interiktalen Spikes mittels realistischem Volumenleiter. Man sieht die EEG-Elektroden, das verwendete Volumenleitermodell (realistisches 3-Grenzflächen-Modell) sowie den zeitlichen und örtlichen Verlauf der rekonstruierten Hirnaktivität.

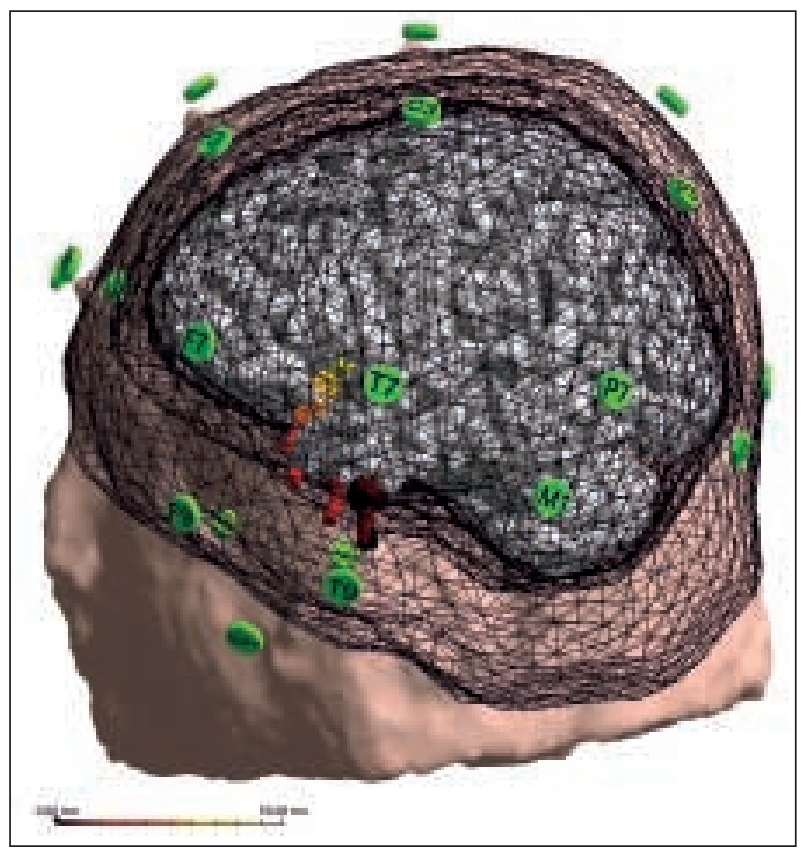

\section{Abbildung 5}

Dieselben Ergebnisse wie in Abb. 4, allerdings ohne Abbildung des Volumenleiters. Man beachte die sehr gute Übereinstimmung zwischen den Ergebnissen der Quellanalyse und der Anatomie; der Aktivitätsverlauf erfolgt entlang des Temporallappens. Solche Ergebnisse können dazu dienen, implantierte Elektroden zielgenau zu plazieren oder in Zukunft einmal ganz ohne sie auszukommen.

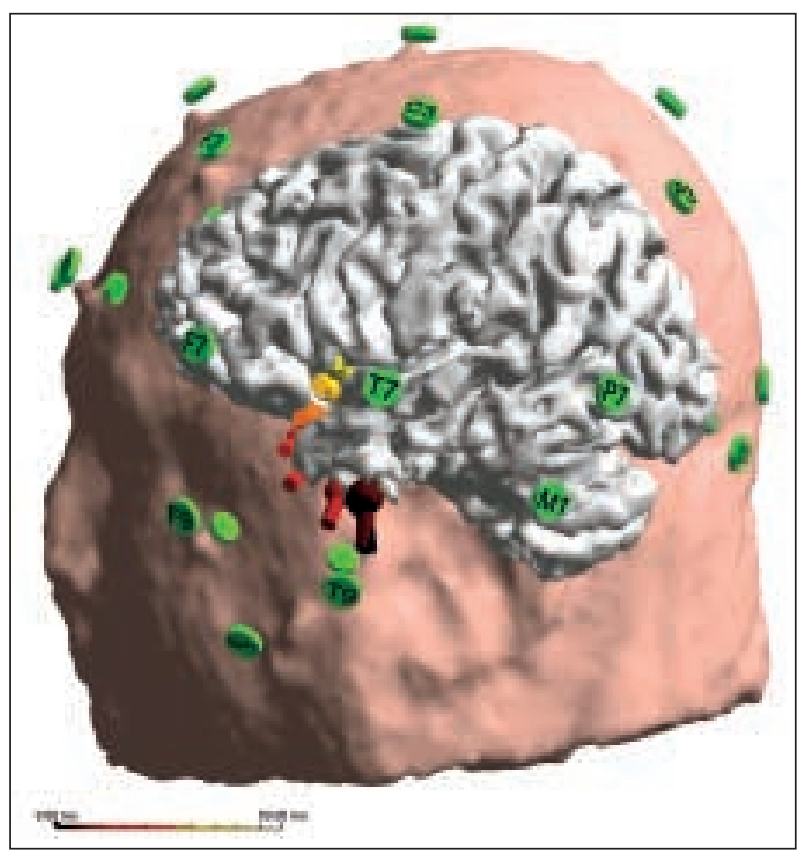

\section{Literatur}

- Foldvary N, Bingaman WE, Wyllie E. Surgical treatment of epilepsy. Neurologic Clinics 2001; 2:491-515.

- Schmidt D. Epilepsien. Fragen und Antworten. München: W. Zuckschwerdt Verlag; 2002.

- Fuchs M, Wagner M, Kastner J. Boundary element method volume conductor models for EEG source reconstruction. Clinical Neurophysiology 2001; 112:1400-7.

- Fuchs M, Wagner M, Köhler Th, Wischmann HA. Linear and nonlinear current density reconstructions. J Clinical Neurophysiol 1999;16:267-95.

- Shorvon S, Dreifuss F, Fish D, Thomas D (eds.). The Treatment of Epilepsy. Cambridge: Blackwell Science Ltd; 1996.

- Wagner M. Rekonstruktion neuronaler Ströme. Aachen: Shaker Verlag; 1998. 